\title{
The importance of anti-transglutaminase IgA antibody detection in the diagnosis of celiac disease - case report of an inappropriate diagnostic approach
}

\author{
Edyta Szymańska, Sylwia Szymańska, Joanna Pawłowska, Ewa Orłowska, Ewa Konopka, Bożena Cukrowska
}

The Children's Memorial Heath Institute, Warsaw, Poland

Prz Gastroenterol 2015; 10 (4): 250-253

DOI: $10.5114 /$ pg.2015.52415

Address for correspondence: Prof. Bożena Cukrowska, The Children's Memorial Heath Institute, 20 Dzieci Polskich Ave. 04-730 Warsaw, Poland, phone: +48 2281510 91, e-mail: b.cukrowska@czd.pl

Celiac disease (CD) is a chronic autoimmune intestinal disease caused by intolerance to gluten [1]. It is characterised by immune-mediated enteropathy, associated with maldigestion and malabsorption of most nutrients and vitamins. The clinical presentations of CD differ depending on the patients' age. Children younger than 2-3 years of age in most cases suffer from classical $\mathrm{CD}$, whereas older children and adults usually present with atypical forms [2, 3]. Until recently, firm diagnosis of CD has been established based on small intestinal biopsy; however, in 2012 the European Society for Paediatric Gastroenterology, Hepatology, and Nutrition (ESPGHAN) re-edited their diagnostic guidelines for CD in children [1]. According to the new recommendations (Table I), the presence of IgA anti-intestinal transglutaminase 2 antibody (TGA-IgA) is the most sensitive serological marker, and detection of those specific antibodies together with determination of total IgA should be the first tests when CD is suspected in symptomatic children.

According to the novel recommendations, the diagnosis of CD without biopsy is allowed in certain cases [4]. Such a diagnostic approach concerns patients who present with clinical CD symptoms and strictly fulfil the following criteria: 1) TGA-IgA are highly elevated (greater than 10 times the upper limit of normal values), 2) antibody positivity is verified by IgA anti-endomysial antibodies (EMA) from a blood sample taken separately from the initial test, 3) positive HLA-DQ2 or/and -DQ8 haplotypes are confirmed by genetic tests, and 4) the response to a gluten-free diet is observed. Nonetheless, histological evaluation of duodenal specimens with the use of modified Marsh-Oberhuber classification is still preferred in other cases, including patients with IgA de- ficiency, in whom only the antibodies in the IgG class are present.

The histological criteria include the number of intraepithelial lymphocytes (IEL) per 100 enterocytes in the small intestine, the presence of crypt hyperplasia, and/or villous atrophy. Increased IEL number is actually considered the most important histological feature indicative of CD because atrophy of small intestinal mucosa with an absence of normal intestinal villi may occur in many entities such as microvillus inclusion disease, autoimmune enteropathy, intolerance to food (e.g. cow's milk, eggs, or soya), and eosinophilic gastroenteritis [5]. Previously, CD was diagnosed with the presence of flattened duodenal villi (grade 3 according to Marsh-Oberhuber scale). The new criteria assume that shortened villi (grade 2) are enough to recognise CD, and the disease should be suspected in symptomatic patients with a significantly increased number of IEL (> 30 per 100 enterocytes) [4]. The new diagnostic criteria emphasise the importance of TGA-IgA determination with a supporting role from EMA. Nonetheless, current recommendations have not always been introduced, which could result in difficulties during the diagnostic process.

We report a case of a challenging diagnosis of $C D$ in an adolescent girl, in whom EMA examination was done as the first serological test.

A 14-year-old girl was admitted to the regional hospital due to chronic diarrhoea persisting for 5 weeks. The physical examination at admission revealed no abnormalities apart from poorly developed subcutaneous tissue - both the body mass and the height at the $3^{\text {rd }}$ percentile. The laboratory tests showed leucocytosis and eosinophilia with increased IgE level. To exclude CD, EMAs were done, and they were negative. The ultra- 
Table I. New ESPGHAN guidelines for the diagnosis of celiac disease in children [4]

\begin{tabular}{|c|c|}
\hline Group of patients & Diagnostic approach \\
\hline Symptomatic patients & $\begin{array}{l}\text { 1. Test for CD-specific antibodies is the first step: } \\
\text { - TGA-IgA from a blood sample and total IgA } \\
\text { - in subjects with either primary or secondary humoral IgA deficiency, at least one additional test measuring } \\
\text { IgG class CD-specific antibodies is recommended, e.g. antibodies against deamidated gluten peptide (DPG) } \\
\text { or TGA-IgG } \\
\text { - if CD antibodies are negative in an IgA-competent symptomatic patient, the diagnosis of CD is unlikely, but } \\
\text { in seronegative cases with severe symptoms and a strong clinical suspicion of CD, and in children younger } \\
\text { than } 2 \text { years, small intestinal biopsies and HLA-DQ testing are recommended } \\
\text { 2. When the level of TGA-lgA is lower than } 10 \text { times the upper limit of normal, small intestine biopsy is } \\
\text { obligatory: } \\
\text { - biopsies should be taken from the second/third portion of the duodenum (at least four samples), and at } \\
\text { least one biopsy should be taken from the duodenal bulb; } \\
\text { - indicative histological features of CD is grade } 2 \text { and } 3 \text { according modified Marh-Oberhuber scale } \\
\text { 3. In the absence of CD-specific antibodies and/or HLA-DQ2 or HLA-DQ8 heterodimers, other causes of } \\
\text { enteropathy must be considered }\end{array}$ \\
\hline $\begin{array}{l}\text { Diagnosis of CD without } \\
\text { duodenal biopsy }\end{array}$ & $\begin{array}{l}\text { 1. In patients with signs or symptoms suggestive of CD and high TGA-IgA titres (levels > } 10 \text { times upper limit } \\
\text { of normal) } \\
\text { 2. Antibody positivity should be verified by EMA-IgA from a blood sample drawn on an occasion separate } \\
\text { from the initial test } \\
\text { 3. The presence of HLA-DQ2 or HLA-DQ8 haplotype }\end{array}$ \\
\hline
\end{tabular}

sound imaging showed no abnormalities. Negative stool cultures excluded gastrointestinal infections. Bearing in mind the above laboratory results, colonoscopy without gastroduodenoscopy was performed. Histological examination showed chronic inflammation in the large intestine with polymorphic infiltrates, in which eosinophils dominated. The girl, with an initial discharge diagnosis of ulcerative colitis (UC), was given mesalazine and she was referred to the Department of Gastroenterology, Hepatology, and Feeding Disorders of the Children's Memorial Health Institute $(\mathrm{CMHI})$ in Warsaw to extend the diagnostic workup. At first, complete serological testing according to the ESPGHAN guidelines for diagnosis of CD was performed (TGA-IgA, total IgA, and EMA, which were previously negative). Total IgA within normal limits, increased TGA-IgA ( $22 \mathrm{U} / \mathrm{ml}$; normal range up to $10 \mathrm{U} / \mathrm{ml}$ ), and slightly positive EMA in titre $1: 5$, but negative in titre $1: 10$, were found. Both gastroduodenoscopy and colonoscopy were performed. Histological examination revealed pathologic changes restricted to the duodenum. Although duodenal villi were normally formed with the absence of inflammation (Figure $1 \mathrm{~A}$ ), the number of IEL was increased, varying from 30 up to 50 lymphocytes per 100 enterocytes (Figure $1 \mathrm{~B}$ ). Thus, histological changes were classified as grade 1 according to the modified Marsh-Oberhuber classification. There were no abnormalities within the large intestine; therefore, the diagnosis of UC was excluded and the treatment with mesalazine was discontinued. The ordinary gluten-full diet was ordered, and 6 months later the girl was re-admitted to the $\mathrm{CMHI}$ in order to perform control gastroduodenoscopy. This time histopathological features were explicitly indicative of CD and classified as grade $3 \mathrm{C}$ according to Marsh-Oberhuber classification. Either in duodenal bulb or in other parts of the duodenum flattened villi with significantly increased number of IEL (50 lymphocytes per 100 enterocytes) and moderate polymorphic infiltrates in the lamina propria were observed (Figure 2). Repeated serological tests showed increased level of TGA-IgA $(16 \mathrm{U} / \mathrm{ml})$ and negative EMA in titre $1: 5$. Finally, CD was recognised. A gluten-free diet and a follow-up visit at the outpatient gastroenterology clinic were ordered. At present, the girl is doing well, the diarrhoea has stopped, and her nutritional status has improved.

We report a case of 14-year-old adolescent girl as an example of an inappropriate approach towards CD diagnostic workout. Although the new ESPGHAN guidelines were established two years ago, problems with their implementation still exist. Our patient was admitted to the regional hospital due to chronic diarrhoea, where, in order to exclude CD, only EMA testing was performed. As a consequence of negative serological results and suspicion of UC, only colonoscopy was performed. In our Institute complete serological screening showed positive TGA-IgA and negative EMA in titre $1: 10$ (we do not know what EMA titre was applied at the regional hospital, but often commercially accepted serum dilution is $1: 10$ ). Many studies confirm that TGA- IgA presents higher sensitivity than EMA [6, 7]. Furthermore, TGA immunoassays are easier to perform, less subjective, and more amenable to automation than EMA im- 

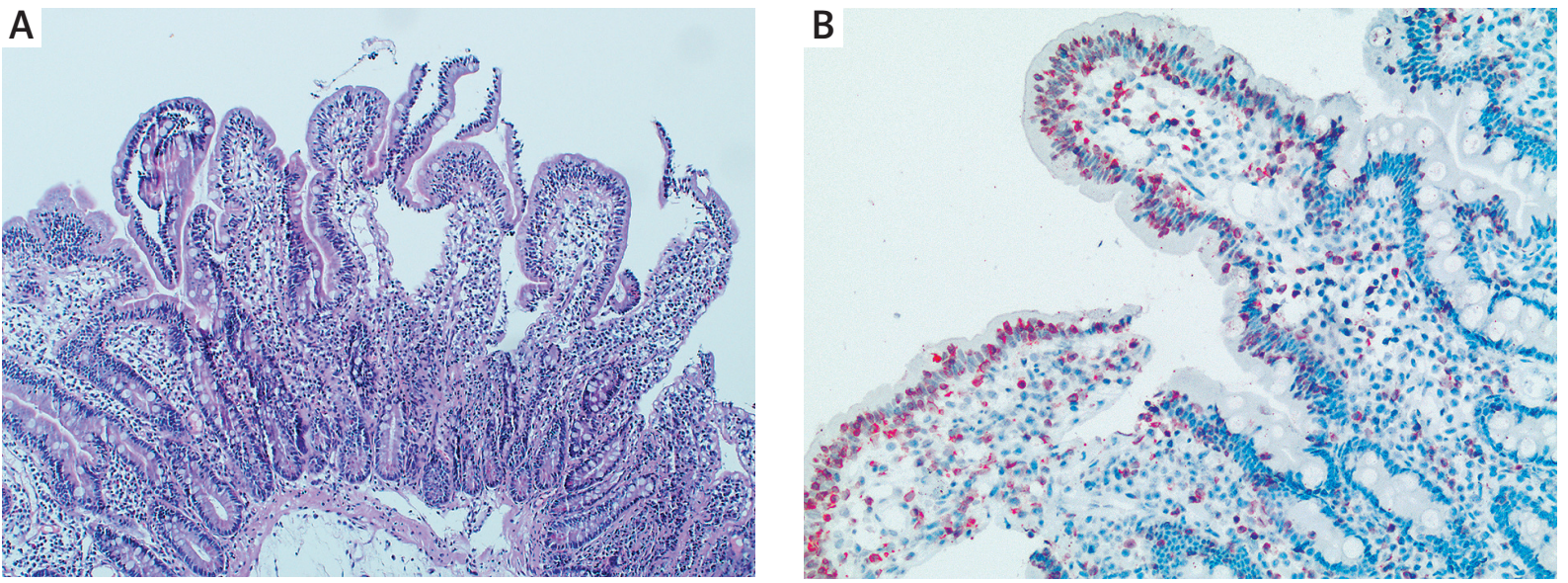

Figure 1. A - The biopsy specimen from the duodenum taken during the first hospitalisation in the Children's Memorial Heath Institute. Duodenal villi are normally formed with the absence of inflammation, the number of IELs is significantly increased, varying from 30 up to 50 lymphocytes per 100 enterocytes. Original magnification 20×. B - Increased number of IEL stained with anti-CD3 antibody (red colour) are visible in the duodenal specimen. Original magnification 40x

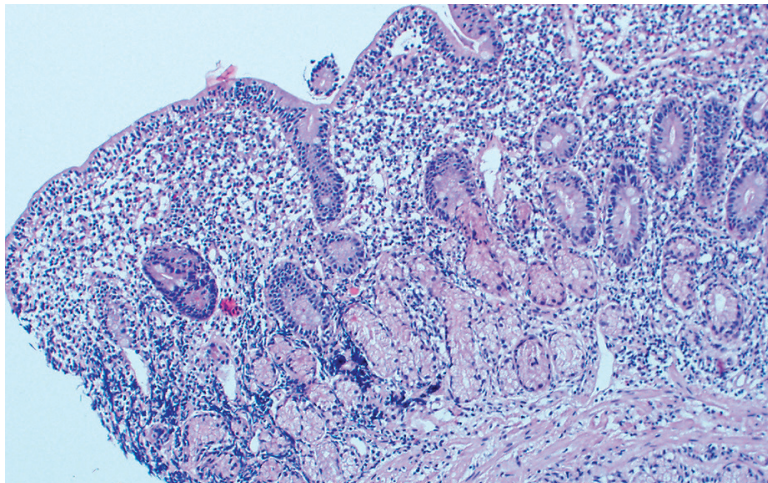

Figure 2. The biopsy specimen from the bulb duodenum taken during the second hospitalisation. Flattened villi and significantly increased IEL number characteristic of active CD are shown. Original magnification $20 x$

munoassays. Thus, anti-TGA tests are more likely to be offered as first-line screening assays in the evaluation of $C D$, not only in paediatric patients, but also in adults [8]. Currently, EMA should be used only in selected cases, when the diagnosis of CD can be made without small intestine biopsy, i.e. in children and adolescents with symptoms suggestive of CD and with high TGA-IgA concentration > 10 times the upper normal limit [4]. In this situation, antibody positivity should be verified by EMA from a blood sample drawn on an occasion separate from the initial test to avoid false-positive serology results owing to mislabelling of blood samples or other technical mistakes. In addition, when CD diagnosis is to be established without histological analysis of duodenal specimens, molecular examination of HLA must be done to confirm HLA-DQ2 and/or HLA-DQ8 haplotypes. Our observations have revealed that in about $20-30 \%$ of cases, CD can be recognised without biopsy. Moreover, during the last 2 years, more and more children have had their diagnosis established in such a way at our Institute. However, in the described girl the biopsy of the small intestine was necessary. The duodenal specimens obtained from the first biopsy showed only an increased number of IEL limited to the duodenum. Thus, the patient could only be diagnosed with so-called potential type of CD (positive specific celiac antibodies and grade 1 according Marsh-Oberhuber scale), but so far there are no clear recommendations whether a gluten-free diet should be introduced in such cases. As our patient had already been treated with anti-inflammatory medications because of suspected colitis, and in order to verify the effect of an ordinary, gluten-full diet on histological features of the duodenum, the endoscopy with biopsy was repeated 6 months later. This time, histological changes finally confirmed CD diagnosis. It is necessary to underline that the histological features in CD are patchy, and develop dynamically depending on either the dose of ingested gluten or the duration of exposure to gluten [9]. Moreover, in some patients they appear only in the duodenal bulb [10]. That is why tissue specimens should be taken preferably during upper endoscopy from the bulb (at least one specimen) and from the second or third portion of the duodenum (all together at least four specimens) [4]. Our case shows that the presence of specific TGA may precede typical histological changes in the small intestine, and mucosal 
abnormalities develop over time when a normal-gluten diet is applied. Thus, in patients with positive specific TGA-IgA antibodies and a lack of specific histological changes, a follow up biopsy should be done. Although in our patient HLA examination was not performed, in such atypical patients, molecular testing could be done due to its great negative predictive value (about 99\% of patients have HLA-DQ2 and/or HLA-DQ8 haplotype) $[11,12]$. Interestingly, the specimens taken during the second colonoscopy presented normal histological features, whereas chronic inflammation with polymorphic and eosinophilic infiltrates had been found in the large intestine when colonoscopy had been done at the regional hospital. It is difficult to explain this phenomenon. However, the hypothesis that CD onset was initiated by inflammatory/allergic reactions to antigens other than gluten cannot be excluded. Although gluten is the main external trigger of $C D$, its intake does not fully explain the pathogenesis of the disease. The disease can develop at any time during life, irrespective of diet - whether including gluten proteins or not. Lahdenperä et al. have proven that a gluten-free diet, which improves mucosal lesions, does not correct the increased activation of pro-inflammatory mediators of CD [13]. Therefore, other environmental factors are thought to be involved in the initiation and progression of pathological processes in CD. Finally, it must be underlined that the whole family of a person with CD should be under medical surveillance due to their falling into a highrisk group for disease development [4]. Our preliminary studies showed that about $6 \%$ of first-degree relatives of CD patients have undiagnosed CD [14]. Unfortunately, CD screening for close relatives of a patient is not covered by the Polish health insurance service.

TGA-IgA antibodies are sensitive and specific markers of CD, and according ESPGHAN guidelines TGA-IgA should be detected as the first serological test when $C D$ is suspected in symptomatic patients with normal IgA levels.

\section{Acknowledgments}

This paper was supported by the projects of the Children's Memorial Health Institute (no. 135/2013 to B.C. and no. $221 / 13$ to P.S).

\section{Conflict of interest}

The authors declare no conflict of interest.

\section{References}

1. Sapone A, Bai JC, Ciacci C, et al. Spectrum of gluten-related disorders: consensus on new nomenclature and classification. BMC Med 2012; 7: 10-3.
2. Mäki M, Kallonen K, Lähdeaho ML, Visakorpi JK. Changing pattern of childhood coeliac disease in Finland. Acta Paediatr Scand 1998; 77: 408-12.

3. Rampertab SD, Pooran N, Brar P, et al. Trends in the presentation of celiac disease. Am J Med 2006; 119: 355e 9-14.

4. Husby S, Koletzko S, Korponay-Szabo IR, et al. ESPGHAN guidelines for diagnosis of coeliac disease. J Paediatr Gastroenrol Nutr 2012; 54: 136-60.

5. Bao F, Green PHR, Bhagat G. An update on celiac disease histopathology and the road ahead. Arch Pathol Lab Med 2012; 136: 735-45.

6. Wengrower D, Doron D, Goldin E, Granot E. Should stored serum of patients previously tested for celiac disease serology be retested for transglutaminase antibodies? J Clin Gastroenterol 2006; 40: 806-8

7. Nakazawa $\mathrm{H}$, Makishima $\mathrm{H}$, Ito $\mathrm{T}$, et al. Screening tests using serum tissue transglutaminase IgA may facilitate the identification of undiagnosed celiac disease among Japanese population. Int J Med Sci 2014; 11: 819-23.

8. Husby S, Murray JA. Diagnosing coeliac disease and the potential for serological markers. Nat Rev Gastroenterol Hepatol 2014; 11: 655-63.

9. Ravelli A, Villanacci V, Monfredini C, et al. How patchy is patchy villous atrophy? Distribution pattern of histological lesions in the duodenum of children with celiac disease. Am J Gastroenterol 2010; 105: 2103-10.

10. Mansfield-Smith S, Savalagi V, Rao N, et al. Including duodenal bulb histology should be standard of care when evaluating celiac disease in children. Pediatr Dev Pathol 2014; 17: 339-43.

11. Szałowska D, Bąk-Romaniszyn L. Case report family recognition of celiac disease. Prz Gastroenterol 2013; 8: 390-5.

12. Szałowska-Woźniak DA, Bąk-Romaniszyn L, Cywińska-Bernas A, Zeman K. Evaluation of HLA-DQ2/DQ8 genotype in patients with celiac disease hospitalised in 2012 at the Department of Paediatrics. Prz Gastroenterol 2014; 9: 32-7.

13. Lahdenperä A, Ludvigsson J, Fälth-Magnusson K, et al. The effect of gluten-free diet on Th1-Th2-Th3-associated intestinal immune responses in celiac disease. Scand J Gastroenterol 2011; 46: 538-49.

14. Cukrowska B, Zagroba M, Konopka E. HLA-DQ2/DQ8 haplotyping and specific antibodies estimation in first line relatives to patients with celiac disease. [Polish]. Przegl Ped 2013; 43 (Suppl. 1): 35.

Received: 28.11.2014

Accepted: 6.01 .2015 